\title{
ONE-SIDED APPROXIMATION AND VARIATIONAL INEQUALITIES
}

\author{
BY UMBERTO MOSCO AND GILBERT STRANG ${ }^{1}$ \\ Communicated by Hans Weinberger, May 1, 1973
}

ABSTRACT. For piecewise linear approximation of the unilateral Laplace equation (also known as the obstacle problem, and governed by a variational inequality), we prove that the gradient of the error $u-u_{h}$ is of order $h$. The proof rests on approximation of nonnegative functions $U$ by nonnegative splines $V_{h} \leqq U$.

We are interested in one of the first and most fundamental of the variational problems introduced by Fichera, Stampacchia, and Lions [3], [4], [6]:

Find that function $u$ in the convex set

which minimizes

$$
K=\left\{v \mid v \in \mathscr{H}_{0}^{1}(\Omega), v \geqq \psi \text { on } \Omega\right\}
$$

$$
I(v)=a(v, v)-2(f, v)=\iint_{\Omega}\left(v_{x}^{2}+v_{y}^{2}-2 f v\right) d x d y .
$$

If the "obstacle function" $\psi$ were absent, this would be the classical Dirichlet problem for Poisson's equation $-\Delta u=f$, and the condition for a minimum would be a variational equation: $a(u, v)=(f, v)$ for $v$ in $\mathscr{H}_{\mathbf{0}}^{\mathbf{1}}$. This is the weak form of Poisson's equation, and coincides with the engineer's "equation of virtual work".

For minimization over $K$ instead of the full space $\mathscr{H}_{0}^{1}$, the variational equation turns into an inequality-just as, for minimization of a function $g$ over $0 \leqq x \leqq 1$, the possibility of minima at the endpoints alters the usual $d g / d x=0$. The condition that $u$ be minimizing is

$$
a(u, v-u) \geqq(f, v-u) \text { for all } v \text { in } K .
$$

Suppose we solve this problem approximately, by the Ritz principle: The approximation $u_{h}$ minimizes the functional I over a finite-dimensional

AMS (MOS) subject classifications (1970). Primary 35J20, 65N30, 41A15.

Key words and phrases. Approximation, splines, variational problem, Ritz method.

1 The first author was supported by GNAFA-CNR and enjoyed the hospitality of the Courant Institute under a CNR-NATO grant. The second author was supported by the National Science Foundation (P22928). 
convex set $K_{h}$. In analogy with (1), this means that $u_{h}$ satisfies

$$
a\left(u_{h}, v_{h}-u_{h}\right) \geqq\left(f, v_{h}-u_{h}\right) \text { for all } v_{h} \text { in } K_{h} .
$$

We want to estimate the error, using the $\mathscr{H}_{\mathbf{0}}^{\mathbf{1}}$ norm $\left\|u-u_{h}\right\|=$ $\left(a\left(u-u_{h}, u-u_{h}\right)\right)^{1 / 2}$ which is intrinsic to the problem. Without $\psi$, such estimates are classical: $u_{h}$ is the projection of $u$ onto $K_{h}$ (which becomes a subspace instead of a general convex set) and $\left\|u-u_{h}\right\|$ is exactly the distance from $u$ to $K_{h}$. The unilateral constraint $u \geqq \psi$ destroys this pattern, and our $u_{h}$ is unlikely to be the projection of $u$.

We shall work with the following example. Let $\Omega$ be a convex polygon, and carve it into triangles of side less than $h$. Let $S_{h}$ be the space of continuous piecewise linear functions on this triangulation, vanishing on $\partial \Omega . S_{h}$ is the subspace of $\mathscr{H}_{0}^{\mathbf{1}}(\Omega)$ which Courant proposed for approximation of the Dirichlet problem; it was the first of the finite element spaces [7]. Suppose $\psi_{h}$ is the linear interpolate of $\psi: \psi_{h}$ agrees with $\psi$ at all vertices of the triangulation, and takes the form $a+b x+c y$ within each triangle. Then we choose $K_{h}=S_{h} \cap\left\{v_{h} \geqq \psi_{h}\right.$ on $\left.\Omega\right\}$. The minimization of $I$ over $K_{h}$-in other words, the computation of $u_{h}$-is numerically not a difficult problem (cf. [5]).

To admit a smooth domain instead of a polygon would simplify the theory for the continuous problem; but it complicates the construction of the discrete subset $K_{h}$. Also, because all the novelty (and difficulty) comes from $\psi$ and not $f$, we shall assume $f=0$. And to keep this note brief, we require that $\psi$ lie on both $\mathscr{H}_{0}^{1}$ and $\mathscr{H}^{2}$. Then it is known (BrézisStampacchia-Lewy) that the solution $u$ also lies in $\mathscr{H}^{2}$, and that its norm can be estimated from the data: $\|u\|_{2} \leqq C\|\psi\|_{2}$.

We first ask how closely such a function can be approached by elements of the convex set $K_{h}$. Suppose we choose the particular element $u_{I}$, the interpolate of $u$, which agrees with $u$ at every vertex of the triangulation (and lies in $K_{h}$ ). Then it is a standard estimate in approximation theory [7] that

$$
\left\|u-u_{I}\right\| \leqq C h\|u\|_{2} .
$$

Although $u_{I}$ may not achieve precisely the minimum distance from $u$ to $K_{h}$, it is at least "quasi-optimal"; $\left\|u-u_{I}\right\|$ is within a constant multiple of this distance, which is of order $h$. Our problem is to show that the Ritz approximation $u_{h}$ is also quasi-optimal, in other words that $\left\|u-u_{h}\right\|=O(h)$.

For variational inequalities in general, this will not be the case. In the plane, consider the problem of minimizing $I=x^{2}+y^{2}$-in other words, of finding the points $u$ in $K$ and $u_{h}$ in $K_{h}$ closest to the origin. If $K$ is the quadrant $x \geqq 1, y \geqq 0$, then obviously $u=(1,0)$. We will have $u_{h}=\left(1, h^{1 / 2}\right)$, 
if $K_{h}$ is formed from $K$ by deleting the small triangle below the line connecting this point $u_{h}$ to $v_{h}=(1+h, 0)$. The distance from $u$ to $K_{h}$ is less than $\left|u-v_{h}\right|=h$, and therefore $\left|u-u_{h}\right|=h^{1 / 2}$ is much too large to be quasioptimal. Aubin [1] has proved that our example illustrates the worst possible case; always $\left\|u-u_{h}\right\|^{2} \leqq c \operatorname{dist}\left(u, K_{h}\right)$.

In the obstacle problem, the first step is to notice that for some elements $v$, equality holds in the variational inequality (1). This will be the case if, together with $v$, also $2 u-v$ lies in $K$. Replacing $v$ by $2 u-v$ in (1), that inequality is reversed-and equality holds. Obviously $u$ is the average of $v$ and $2 u-v$; our observation is simply that if $u$ is not an extreme point of the convex set $K$, then there are directions in which $u$ is interior to a line segment, and in these directions the usual equality holds.

Let the cones $C$ and $C_{h}$ be composed of the nonnegative functions in $\mathscr{H}_{0}^{1}$ and its subspace $S_{h}$, respectively. Thus $U=u-\psi$ lies in $C$, and $U_{h}=$ $u_{h}-\psi_{h}$ in $C_{h}$.

Lemma. If $V_{h} \in C_{h}$ and $2 U-V_{h} \in C$, then

$$
\left\|u-u_{h}\right\| \leqq\left\|\psi-\psi_{h}\right\|+\left\|U-V_{h}\right\| .
$$

Proof. Since both $v=\psi+V_{h}$ and $2 u-v=\psi+\left(2 U-V_{h}\right)$ lie in $K$, equality must hold in (1): with $f=0$, this means that

$$
a(u, v-u)=a\left(u, V_{h}-U\right)=0 .
$$

Choosing $v_{h}=\psi_{h}+V_{h}$ in (2), we also have

$$
a\left(u_{h}, v_{h}-u_{h}\right)=a\left(u_{h}, V_{h}-U_{h}\right) \geqq 0 .
$$

Finally, with $v=\psi+U_{h}$ in (1),

$$
a\left(u, U_{h}-U\right) \geqq 0 .
$$

It follows from (5)-(7) that $a\left(u-u_{h}, U_{h}-V_{h}\right) \geqq 0$. Therefore

$$
\begin{aligned}
\left\|u-u_{h}\right\|^{2} & =a\left(u-u_{h}, u-u_{h}\right) \\
& =a\left(u-u_{h}, \psi-\psi_{h}\right)+a\left(u-u_{h}, U-U_{h}\right) \\
& \leqq a\left(u-u_{h}, \psi-\psi_{h}\right)+a\left(u-u_{h}, U-V_{h}\right) \\
& \leqq\left\|u-u_{h}\right\|\left(\left\|\psi-\psi_{h}\right\|+\left\|U-V_{h}\right\|\right) . \quad \text { Q.E.D. }
\end{aligned}
$$

Since $\psi_{h}$ is the interpolate of $\psi$, we know from (3) that $\left\|\psi-\psi_{h}\right\| \leqq$ $C h\|\psi\|_{2}$. To use the lemma we have also to find a $V_{h}$ in $C_{h}$ which is close in norm to $U$, and everywhere below $2 U$. In fact, we shall be able to keep $V_{h} \leqq U$-leading to one-sided approximation of a nonnegative $U$ by a nonnegative linear spline $V_{h}$. 
THEOREM. Suppose that $U \geqq 0$ in the plane polygon $\Omega$, and that $U$ lies in $\mathscr{H}_{0}^{1} \cap \mathscr{H}^{2}$. Then there exists a $V_{h}$ in $S_{h}$ which satisfies

$$
0 \leqq V_{h} \leqq U \text { in } \Omega
$$

and achieves the optimal order of approximation

$$
\left\|U-V_{h}\right\| \leqq C h\|U\|_{2} .
$$

REMARK. We cannot choose $V_{h}$ to be the interpolate $U_{I}$, since this choice may violate $V_{h} \leqq U$. Nor can we subtract a small constant from the interpolate, to keep it below $U$; the condition $V_{h} \geqq 0$ intervenes.

SKETCH OF PROOF. The constraint (8) is satisfied on some subset of $S_{h}$-nonempty, because it contains the zero function. Our choice $V_{h}$ will be any maximal element of this subset.

The proof of (9) would be easy for piecewise linear functions of one variable. At a typical node $x_{j}$, the value of $V_{h}$ cannot be increased while the other nodal values are kept fixed (since $V_{h}$ is maximal). Therefore, either

(i) $V_{h}=U$ at the node $x_{j}$, or

(ii) at some point $\xi$ in $\left[x_{j-1}, x_{j}\right)$ or $\left(x_{j}, x_{j+1}\right], V_{h}=U$ and $V_{h}^{\prime}=U^{\prime}$. In the latter case, with $V_{h}$ tangent to $U$ at $\xi$, it is easy to prove that

$$
\left|U\left(x_{j}\right)-V_{h}\left(x_{j}\right)\right|^{2} \leqq c h^{3} \int_{x_{j-1}}^{x_{j+1}}\left|U^{\prime \prime}\right|^{2} d x
$$

This means that $V_{h}$ is close to the interpolate $U_{I}$, for which (9) is known to be true. In fact, (10) gives an estimate of $U_{I}-V_{h}$ at each node, and therefore of its slope over each interval. Applying the triangle inequality to $U-V_{h}=U-U_{I}+U_{I}-V_{h}$, (9) is proved.

The proof in two dimensions is much more technical, since we cannot speak about tangency at $\xi$; the assumption $U \in \mathscr{H}^{2}$ does not imply differentiability at a point. Nevertheless the theorem continues to hold, and will be published by the second author in the Proceedings of the Symposium on Computing Methods (IRIA, France, 1973). The theorem appears to extend also to three dimensions, but not to $R^{5}$-where De Giorgi has shown us a nontrivial function $u \geqq 0$ which vanishes on a dense set, forcing $v_{h}=0$. The order of one-sided approximation by splines of higher degree is an open question even in $R^{1}$.

The estimate we hoped for-that $\left\|u-u_{h}\right\| \leqq C h\|\psi\|_{2}$-follows immediately from the lemma and theorem. We have just received from Richard Falk [2] another proof of this estimate. And we understand that Baiocchi has some preliminary results about convergence of the free boundary (separating $U=0$ from $U>0$ ). 


\section{REFERENCES}

1. J. P. Aubin, Approximation of variational inequalities, Functional Analysis and Optimization, E. R. Caianiello (Editor), Academic Press, New York, 1966, pp. 7-14. MR 35 \#4743.

2. R. Falk, Error estimates for the approximation of a class of variational inequalities (preprint).

3. G. Fichera, Boundary value problems of elasticity with unilateral constraints, Handbuch der Physik, Bd. VI a/2, Springer-Verlag, Berlin, 1972.

4. J. L. Lions and G. Stampacchia, Variational inequalities, Comm. Pure Appl. Math. 20 (1967), 493-519. MR 35 \#7178.

5. U. Mosco and F. Scarpini, Complementarity systems and approximation of variational inequalities (to appear).

6. G. Stampacchia, Formes bilinéaires coercitives sur les ensembles convexes, C.R. Acad. Sci. Paris 258 (1964), 4413-4416. MR 29 \#3864.

7. G. Strang and G. Fix, An analysis of the finite element method, Prentice-Hall, Englewood Cliffs, N.J., 1973.

Instituto di Calcolo della Probabilità, University of Rome, Rome, italy

Department of Mathematics, Massachusetts institute of Technology, CAMBridge, MassachusetTs 02139 